\title{
Solar Flares as Cascades of Reconnecting Magnetic Loops
}

\author{
D. Hughes, ${ }^{1}$ M. Paczuski, ${ }^{1}$ R.O. Dendy ${ }^{2}$ P. Helander, ${ }^{2}$ and K.G. McClements ${ }^{2}$ \\ ${ }^{1}$ Department of Mathematics, Imperial College of Science, Technology, and Medicine, London, SW $2 B Z, U K$ \\ ${ }^{2}$ UKAEA, Culham Science Centre, Abingdon, Oxfordshire OX14 3DB, UK
}

(October 29, 2018)

\begin{abstract}
A model for the solar coronal magnetic field is proposed where multiple directed loops evolve in space and time. Loops injected at small scales are anchored by footpoints of opposite polarity moving randomly on a surface. Nearby footpoints of the same polarity aggregate, and loops can reconnect when they collide. This may trigger a cascade of further reconnection, representing a solar flare. Numerical simulations show that a power law distribution of flare energies emerges, associated with a scale free network of loops, indicating self-organized criticality.
\end{abstract}

PACS numbers: 05.65.+b, 52.35.Py, 96.60.Pb, 96.60.Rd

One can think of the magnetic field beneath the photosphere of the sun as a giant ball of yarn. Magnetic flux tubes are irregularly expelled into the corona as loops anchored to the photosphere. They form the building blocks for larger scale magnetic structures such as active regions or the magnetic carpet. A solar flare is a rapid change in a strong, complicated coronal magnetic field [1]. According to a physical picture proposed by Parker, turbulent plasma flow below the photospheric surface drives the anchored flux tubes into complex, stressed configurations [2]. When local magnetic field gradients become sufficiently steep, a plasma instability allows the coronal magnetic field to change its topology via reconnection, suddenly releasing energy [3].

The observed statistics of peak X-ray flux distributions [4], the energy released [5], and the quiescent time intervals between solar flares $[6,7]$ are all characterized by power law distributions. The energy distribution is particularly striking, exhibiting scale-free behavior over more than eight decades in energy [5]. These statistics indicate [8] that the solar corona may be in a state of selforganized criticality (SOC) [9]. Such a view implies that large classical X-ray flares and small transient brightenings in the EUV spectrum are not fundamentally different; they are large and small avalanches of reconnection events. In fact, the statistics of solar coronal behavior shares some common features with other intermittent scale free phenomena such as earthquakes, forest fires, traffic, evolution, and turbulence $[10,11]$.

Here we introduce a dynamical model of multiple magnetic loops that are randomly driven at their footpoints, and can interact. A pair of footpoints, of opposite polarity, anchors each directed loop to a two dimensional surface, representing the photosphere. Footpoints of the same polarity interact by local aggregation on the surface. Loops also interact by exchanging footpoints when they collide. A single rewiring, or reconnection, can lead to more loop collisions and thereby trigger a cascade of further reconnection. These cascades of magnetic loop reconnection are identified with solar flares.
Loop configurations observed in numerical studies of the model (see Fig. 1) are qualitatively similar to the magnetic carpet deduced from observations of the quiet sun $[1,3,12]$. The pattern of loops dynamically forms a scale free network $[13,14]$, where the number of loops emerging from each footpoint is distributed as a power law. This network gives rise to a power law distribution of flare energies. Results from our model also suggest that the probability distribution of net magnetic flux emerging from small areas of the photosphere exhibits power law behavior, while the distribution of lengths of flux tubes in the corona is exponential. Our results support the idea that solar flare statistics reflect coronal SOC resulting from magnetic reconnection.

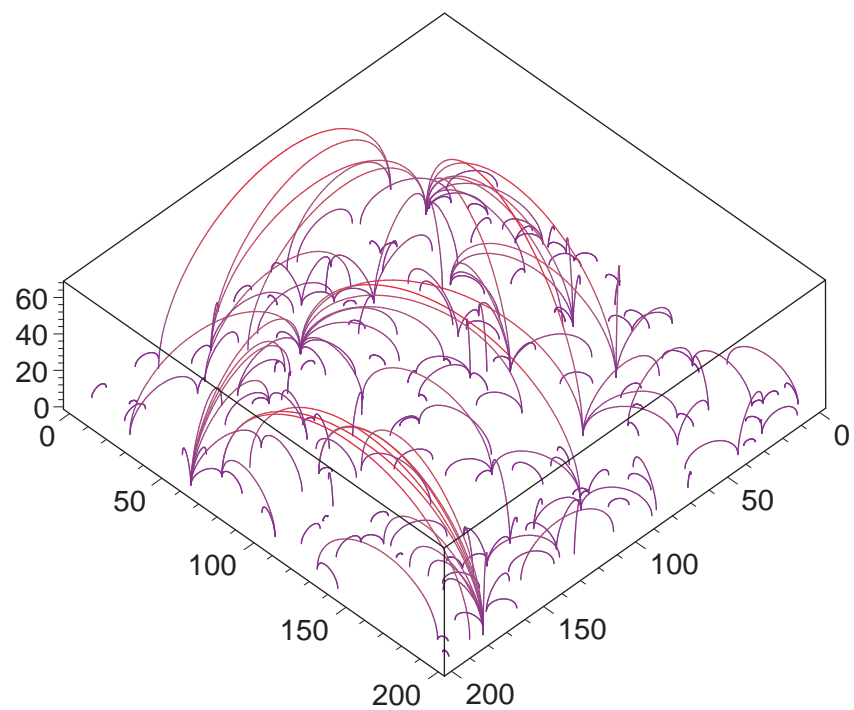

FIG. 1. Snap shot of a configuration of loops in the steady state with $L=200$ and $m=1$ (see text).

The conventional techniques of plasma physics [3] have generated substantial progress in the theoretical understanding and computational modelling of local reconnection in laboratory, space, and solar plasmas. However, it remains computationally prohibitive to use the equations 
of plasma physics to describe extended spatial regions containing multiple evolving flux tubes that interact via magnetic reconnection. This has motivated the adoption of simpler numerical approaches.

$\mathrm{Lu}$ and Hamilton ( $\mathrm{LH})$ used a cellular automaton as a simple model [8] for the reconnection process leading to flares. The LH avalanche model is similar to the original sandpile model of Bak, Tang, and Weisenfeld [9]. It is conceptually far reaching, and has stimulated many further investigations (see for example $[15,16]$ ), as well as other cellular automata models [17].

The multiloop reconnection model introduced here is not a cellular automaton and differs fundamentally from previous approaches. But it makes explicit contact with the physics of solar flares in two ways. First, it retains the basic topological constraints that the high conductivity of the corona and the physics of reconnection impose on magnetic field evolution. Second, it incorporates turbulent footpoint motion and the resultant driving of coronal magnetic field energy. The key elements of our model are as follows:

1. Loop structure. A loop labels the midline of a magnetic flux tube, which has a diameter of order $100 \mathrm{~km}$ at its footpoint on the photosphere [2]. Therefore, the coronal magnetic field is represented by numerous infinitesimally thin directed loops, which are semicircles emerging from the $(x y)$ plane. Each loop has a positive footpoint, where magnetic flux emerges from the photosphere, and a negative one, where flux returns. The size of the system in the $(x y)$ plane, which represents a region of the photospheric surface, is $L \times L$. Loops are labeled by an integer, $n$, and the positions of the two footpoints of the $n$th loop are labeled in the $(x y)$ plane by $\mathbf{r}_{n}^{+}$and $\mathbf{r}_{n}^{-}$. Each loop is associated with one unit of magnetic flux, so that the magnetic energy of a loop is proportional to its length $l_{n}=\frac{\pi}{2}\left|\mathbf{r}_{n}^{+}-\mathbf{r}_{n}^{-}\right|$. The sum of the lengths of the loops, $E=\sum_{n} l_{n}$, is then a measure of the total magnetic energy of the system, and changes in the value of $E$ correspond to the magnetic energy release in solar flares.

2. Footpoint motion. Footpoints are considered to be passively convected by the turbulent plasma motion beneath the photospheric surface [2]. This is represented in the present model by a random walk. At an update step, an arbitrary footpoint is chosen at random and its position is moved, $\mathbf{r} \rightarrow \mathbf{r}+\Delta \mathbf{r}$. The vector $\Delta \mathbf{r}$ has length and angle chosen randomly from uniform distributions between 0 and 1 , and 0 and $2 \pi$, respectively. If the initial loop lengths are small, random footpoint motion will tend on average to increase the length of the loops. In this way, photospheric turbulence pumps magnetic energy into the coronal magnetic field. Note that footpoints can have more than one loop attached, as per step 5 .

3. Loop injection and submergence. The coronal magnetic field is also driven by injection of new loops from beneath the photospheric surface $[1,2]$. In the model, small loops are injected into the system at random locations, with footpoints initially separated by a distance $l_{n l}=4$. Loops with footpoints closer than distance $l_{\min }=2$ are removed from the system. The precise length scales of these two processes do not effect the critical properties of the system. The essential feature is that at small length scales the model dynamically maintains a flow of loops. Thus the magnetic field of the corona is represented as an open system.
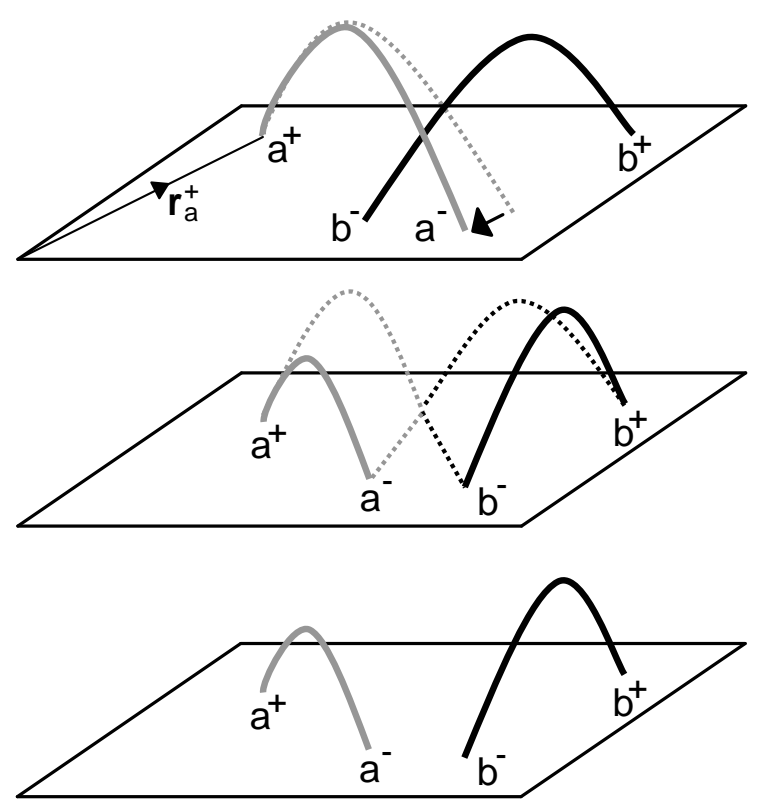

FIG. 2. Diagram showing the process of a reconnection event, from top to bottom. In frame 1 loop a moves from its previous position (dashed line) and crosses loop $b$. In frame 2 the loops exchange footpoints and move to their rewired state. Frame 3 shows the final relaxed configuration.

4. Loop reconnection. Flux freezing in the coronal plasma is relaxed when local field gradients become sufficiently steep: the magnetic field then changes its topology, and releases energy by reconnection events. Reconnection can occur in our model when either (a) two loops collide in three dimensional space, or (b) two footpoints annihilate as explained in step 6. In the first case, the midlines of two flux tubes have crossed resulting in a strong magnetic field gradient at that point. The flux emerging from the positive footpoint of one of the reconnecting loops is then no longer constrained to end up at the other footpoint of the same loop, but may instead go to the negative footpoint of the other loop (see Fig. 2 ). Reconnection is only allowed if it shortens the combined length of the two colliding loops. This process is rapid compared to the driving, because information is transmitted along the flux tubes at the Alfvén speed.

If rewiring occurs, it may happen that one or both loops need to cross some other loop in order to reach its rewired state. Thus a single reconnection between a pair 
of loops can trigger an avalanche-like cascade of causally related reconnection events. The reconnection dynamics of multiply connected footpoints that we used in the numerical simulations is a straightforward extension [18].

5. Footpoint aggregation. It has been observed that footpoints of the same polarity can merge when they approach [2]. In addition, calculations of the coronal magnetic field, based on measurements of the photospheric field, indicate that flux tubes starting at a particular footpoint can terminate at several different footpoints of opposite polarity $[1,3,12]$. In our model, if a footpoint moves within the distance $l_{\text {min }}$ of another footpoint of the same polarity, it is reassigned to the latter footpoint's position and they move as one footpoint thereafter.

6. Footpoint annihilation. Footpoints of opposite polarity, belonging to different flux tubes, can annihilate when they approach on the photosphere [3]. When footpoints of opposite polarity belonging to different loops approach within a distance $l_{\min }$ in the model, both footpoints are eliminated and the remaining two footpoints are attached, forming one loop, where before there were two. The annihilation dynamics of footpoints with more than one attached loop can be implemented in different ways [18]. The scaling behavior of the model does not appear to be sensitive to the precise algorithm. Footpoint annihilation may cause collisions between loops leading to further reconnection, as per step 4 .

7. Time stepping and boundary conditions. In each time step of the model, either a footpoint is chosen for random motion or a new loop is added. This is governed by a control parameter, the stirring rate $m$, such that the number of footpoint updates that separate an update step where a new loop is injected into the system is $m$ times the number of footpoints in the system at that time. We study a system with open reflective boundary conditions; if a footpoint attempts to move outside the $L \times L$ box in the $(x y)$ plane, it is elastically reflected back into the box.

The configuration of loops slowly evolves in response to the driving, aggregation and reconnection processes described above. It reaches a dynamic equilibrium whose statistical character is independent of the initial conditions. Below, numerical simulation results are presented for system size $L=200$ with a range of stirring rates $m$ from 0.01 to 1 , including approximately $10^{7}$ avalanches of reconnection events in the steady state. The observed power law behavior is robust on varying the stirring rate, even though the total magnetic energy and the fluctuations in the total energy vary widely.

Figure 3 shows the probability distribution of the number $k$ of loops emerging from each footpoint, $P_{\text {foot }}(k)$. It is well described by a power law $P_{\text {foot }}(k) \sim k^{-\gamma}$, with $\gamma=2.0 \pm 0.1$ over more than two decades in number of links. The cutoff in the distribution increases with decreasing $m$ for fixed $L$. This is due to the fact that the total number of loops in the system is increasing.

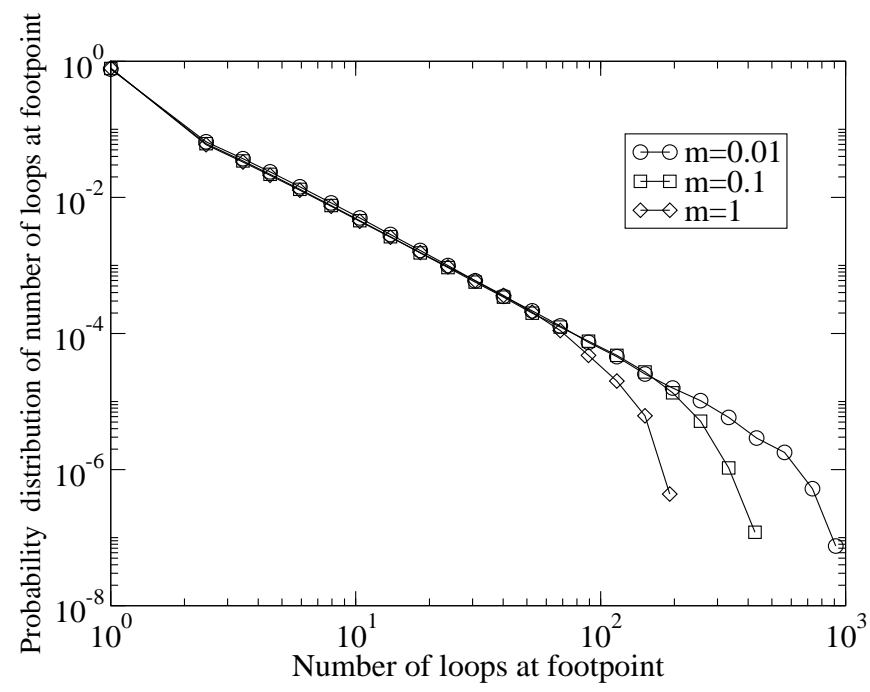

FIG. 3. Distribution of number of loops (links) per footpoint (node). The slope on the log-log scale is -2 . Thus $P_{\text {foot }}(k) \sim k^{-\gamma}$ with $\gamma=2$.

Thus, the system of loops that emerges forms a scale free network, with a cutoff determined only by the number of loops (links) in the system. Scale free networks, in the form of graphs, have been invoked recently to describe a wide variety of phenomena including e.g. citation networks, the internet, and some biological systems; for reviews see Refs. [13,14]. Unlike most of these examples, our network is embedded in three-dimensional space, and geometrical constraints are crucial to the dynamical selforganization by which this network emerges.

Figure 4 shows the probability distribution for flare event energy release, $P_{\text {flare }}(E)$. This also follows a power law $P_{\text {flare }}(E) \sim E^{-\alpha_{E}}$, with $\alpha_{E}=3.0 \pm 0.2$. This value is much larger than the exponent obtained from the $\mathrm{LH}$ type avalanche models, which give $\alpha_{E} \simeq 1.4$ [8]. The distribution of individual loop lengths in our multiloop model, unlike that of flare energies, is exponential.

The total energy released in a solar flare is not directly measured, but is inferred from narrow frequency band observations of photon fluxes. A number of simplifying assumptions are used to relate these fluxes to total energy release, as explained in e.g. Refs. $[15,19,20]$. There is some uncertainty in the value of the exponent of the measured energy distribution of solar flares, with reported values ranging from $\alpha_{E}=1.5$ to $2.6[15,19]$. However, if a consistent set of geometrical assumptions are made, the upper value may be reduced from 2.6 to approximately 2.1 [20]. Our present result is outside this range, but is nevertheless consistent with Parker's conjecture for coronal heating [2] by nanoflares which minimally requires that $\alpha_{E} \geq 2[1,5,15]$.

Another, possibly related difference from observations concerns temporal correlations. Solar flares, like earthquakes and other intermittent phenomena, exhibit power law statistics for the waiting times between events whose 
magnitude exceeds some threshold $[6,7,21]$. The model presented here, like LH avalanche models, does not exhibit any temporal correlations in the waiting times between events. This may be due to the assumption of complete randomness in the external driving.

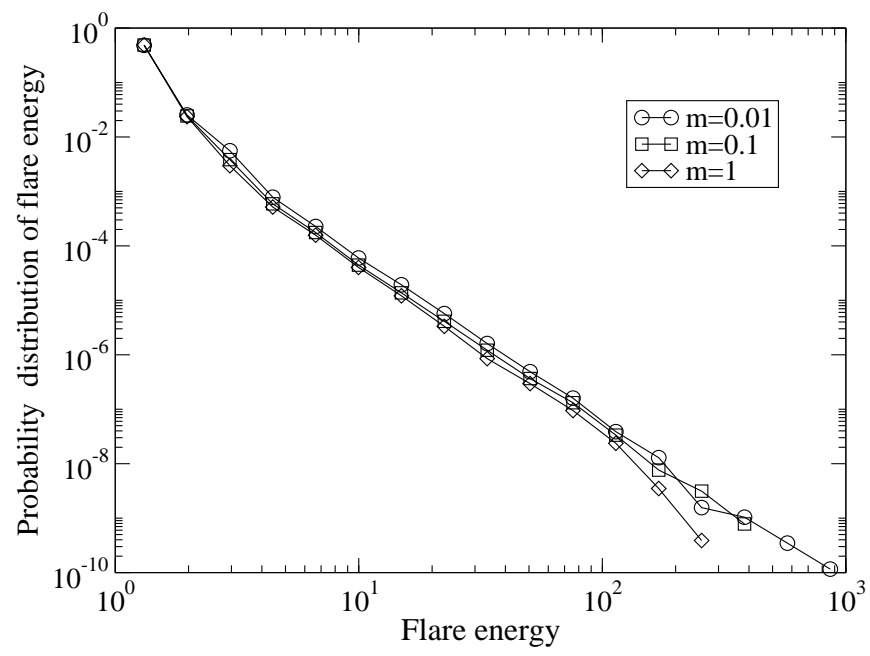

FIG. 4. Distribution of flare energies in the multiloop reconnection model. The slope on the log-log scale is -3 .

Flux tubes are tangled into complex configurations by the turbulent plasma below the photosphere [2]. As a starting point, we have modeled the turbulent forces as inducing both completely random footpoint motion and completely random injection of new loops. These assumptions neglect some features of the photosphere that involve spatiotemporal correlations, such as granulation of the surface, phenomena such as homologous flares, and the observation that active regions, where most large flares occur, have very high rates of magnetic flux emergence compared to the quiet sun [1]. Since we describe the magnetic loop configurations as being passively driven by an external source, our model could easily be extended to incorporate specific spatiotemporal correlations in the driving, in particular in the rate that loops are added to the system at different places.

Following a suggestion by Wheatland [7] that nonstationary driving could induce waiting time correlations, Norman et al have studied LH avalanche models with a driving rate modulated by a random walk and found both a change from exponential to a power law distribution of waiting times, as well as a small change in the critical index $\alpha_{E}$ [19]. Perhaps a similar nonstationary driver would have comparable effects in our model.

We have introduced a model of the solar coronal magnetic field consisting of multiple reconnecting magnetic loops. We believe that this model captures essential elements of the physics governing such structures. Our approach applies to situations involving more loops, and over greater length and time scales, than are accessible to traditional analytical and computational techniques based on the underlying equations.

The striking feature of our multiloop reconnection model is the dynamical self-organization of a magnetic field which gives rise to a power law distribution of solar flare energies, and which forms a scale free network that qualitatively resembles the actual coronal magnetic field. The behavior, $P_{\text {foot }}(k) \sim k^{-\gamma}$, could perhaps be tested by imposing a fine grid on the photosphere, depending on resolution capabilities, and measuring the probability distribution of total magnetic flux within each grid cell. Our model results suggest that this distribution would have power law behavior, with index $\gamma$, while the distribution of lengths of flux tubes would be exponential.

We thank P. Cargill and S.C. Cowley for discussions. This work was funded in part by the UK Department of Trade \& Industry and the EPSRC.

[1] For a review see J.B. Zirker, Journey from the Center of the Sun (Princeton University Press, Princeton, 2002).

[2] E.N. Parker, Astrophys. J. 264, 642 (1983); Astrophys. J 330, 474 (1988); Astrophys. J. 390, 290 (1992); Spontaneous Current Sheets in Magnetic Fields (Oxford University Press, New York, 1994).

[3] E. Priest and T. Forbes, Magnetic Reconnection (Cambridge University Press, Cambridge, 2000).

[4] B.R. Dennis, Solar Phys. 100, 465 (1985).

[5] M.J. Aschwanden et al., Astrophys. J. 535, 1047 (2000).

[6] G. Boffetta et al, Phys. Rev. Lett. 83, 4662 (1999).

[7] M.S. Wheatland, Astrophys. J. Lett. 536, L109 (2000).

[8] E.T. Lu and R.J. Hamilton, Astrophys. J. 380, L89 (1991).

[9] P. Bak, C. Tang, and K. Wiesenfeld, Phys. Rev. Lett. 59, 381 (1987).

[10] P. Bak, How Nature Works (Copernicus, New York, 1996).

[11] D.L. Turcotte, Rep. Prog. Phys. 62, 1377 (1999).

[12] http://www.lmsal.com/carpet.htm

[13] A.-L. Barabási, Linked: The New Science of Networks (Perseus, Cambridge, 2002).

[14] S.H. Strogatz, Nature 410, 268 (2001).

[15] P. Charbonneau et al, Sol. Phys. 203, 321 (2001).

[16] H. Isliker, A. Anastasiadis, and L. Vlahos, Astron. Astrophys. 363, 1134 (2000); Astron. Astrophys. 377, 1068 (2001); M.K. Georgoulis and L. Vlahos, Astron. Astrophys. 33, 721 (1998); A.L. MacKinnon and K.P. Macpherson, Astron. Astrophys. 310, L9 (1996).

[17] J.B. Zirker and F.M. Cleveland, Solar Phys. 145, 119 (1993).

[18] A complete description of the model is contained in D. Hughes et al, in preparation.

[19] J.P. Norman et al, Astrophys. J 557, 891 (2001).

[20] S.W. McIntosh and Charbonneau, Astrophys. J. Lett. 563, L165 (2001); S.W. McIntosh et al, Phys. Rev. E 65, 046125 (2002). 
[21] P. Bak et al, Phys. Rev. Lett. 88, 178501 (2002). 